\title{
Volumetric MR-Guided High-Intensity Focused Ultrasound with Direct Skin Cooling for the Treatment of Symptomatic Uterine Fibroids: Proof-of-Concept Study
}

\author{
Marlijne E. Ikink, ${ }^{1}$ Johanna M. M. van Breugel, ${ }^{1}$ Gerald Schubert, ${ }^{2}$ Robbert J. Nijenhuis, ${ }^{1}$ \\ Lambertus W. Bartels, ${ }^{3}$ Chrit T. W. Moonen, ${ }^{3}$ and Maurice A. A. J. van den Bosch ${ }^{1}$ \\ ${ }^{1}$ Department of Radiology, University Medical Center Utrecht, Heidelberglaan 100, 3584 CX Utrecht, The Netherlands \\ ${ }^{2}$ Philips Healthcare, Philips Medical Systems MR, Äyritie 4, 01511 Vantaa, Finland \\ ${ }^{3}$ Department of Radiology and Image Sciences Institute, University Medical Center Utrecht, Heidelberglaan 100, \\ 3584 CX Utrecht, The Netherlands
}

Correspondence should be addressed to Marlijne E. Ikink; m.ikink@umcutrecht.nl

Received 11 July 2014; Accepted 18 August 2014

Academic Editor: Trevor Andrews

Copyright ( 2015 Marlijne E. Ikink et al. This is an open access article distributed under the Creative Commons Attribution License, which permits unrestricted use, distribution, and reproduction in any medium, provided the original work is properly cited.

Objective. To prospectively assess the safety and technical feasibility of volumetric magnetic resonance-guided high-intensity focused ultrasound (MR-HIFU) ablation with direct skin cooling (DISC) during treatment of uterine fibroids. Methods. In this proof-of-concept study, eight patients were consecutively selected for clinical MR-HIFU ablation of uterine fibroids with the use of an additional DISC device to maintain a constant temperature $\left(T \approx 20^{\circ} \mathrm{C}\right)$ at the interface between the HIFU table top and the skin. Technical feasibility was verified by successful completion of MR-HIFU ablation. Contrast-enhanced T1-weighted MRI was used to measure the treatment effect (nonperfused volume (NPV) ratio). Safety was evaluated by recording of adverse events (AEs) within 30 days' follow-up. Results. All MR-HIFU treatments were successfully completed in an outpatient setting. The median NPV ratio was 0.56 (IQR [0.27-0.72]). Immediately after treatment, two patients experienced coldness related discomfort which resolved at the same day. No serious (device-related) AEs were reported. Specifically, no skin burns, cold injuries, or subcutaneous edema were observed. Conclusion. This study showed that it is safe and technically feasible to complete a volumetric MR-HIFU ablation with DISC. This technique may reduce the risk of thermal injury to the abdominal wall during MR-HIFU ablation of uterine fibroids. This trial is registered with NTR4189.

\section{Introduction}

Over the last decade, minimally or noninvasive treatment options have gained popularity and continue to evolve and expand with developments in technology and with growing experience. Numerous technological advances have been driven by the observed benefits of the minimally invasive approach, including less side effects, shorter recovery time, and favorable cosmetic results. Since the first feasibility report in 2003 [1], magnetic resonance-guided high-intensity focused ultrasound (MR-HIFU) has been successfully employed to treat symptomatic uterine fibroids in a clinical setting. Although not all uterine fibroids are symptomatic, they are in at least $25 \%$ of the Caucasian women in their reproductive years associated with significant morbidity, including abnormal menstrual bleeding, pelvic discomfort, and reproductive dysfunction [2,3]. An increasing number of symptomatic patients demand less invasive treatment methods in order to achieve symptom relief and a better quality of life. MR-HIFU offers the advantage to perform completely noninvasive thermal ablation because the ultrasound transducer is located outside the abdomen and steers high-intensity focused ultrasound energy into the targeted area through the intact skin.

Since 2010, a volumetric MR-HIFU system has been available for routine clinical treatments of uterine fibroids [4]. 
The volumetric ablation approach utilizes the accumulation of heat by electronically steering the focus along outwardmoving concentric circles, producing well-defined regions of protein denaturation, irreversible cell damage, and coagulative necrosis. However, the treatment of larger ablation volumes requires more thermal energy which may lead to a temperature increase along the ultrasound beam axis in the near field (i.e., intermediate layers located between the ultrasound transducer and the target region, such as epidermis, dermis, subcutaneous tissue, and deeper abdominal layers) [5]. During periods of thermal stress, the rate of heat transfer through the skin surface depends primarily on the heat capacity (or ability to absorb heat) and the thermal conductivity (or ability to transfer heat) of the skin to facilitate heat loss [6]. This heat flux may be enhanced through blood circulation by carrying the heat to adjacent tissues $[7,8]$. Temperature rise within the skin layers will mainly occur in the subcutaneous tissue due to its lower specific heat capacity [6], insulator properties $[6,7,9]$, and its lower blood supply [10-12] than that of other tissues in the abdominal wall. Additionally, reflections at boundaries between different media (e.g., air-skin and/or skin-fat) can occur because of differences in the acoustic impedance of various media [13]. The transmission losses from reflection at the skin interface and attenuation through the skin layers might lead to hot spots and skin overheating. Although MR-HIFU ablation of uterine fibroids is related to a low complication rate, skin toxicity and abdominal discomfort have been described by several groups $[1,14-21]$. Undesired heat accumulation in the near field and target area is moderated by enforcing conservative cooling times between the subsequent energy depositions (sonications) to prevent irreversible thermal tissue damage [22, 23], such as skin burns, subcutaneous edema formation, or fat necrosis [24]. The cooling time is chosen to ensure return of the heated tissue layers to body temperature, and cooling ranges typically a few minutes per energy delivery. This leads to undesirably long delays between the sonications, which contributes to prolonged overall treatment times. It would therefore be valuable to regulate the temperature of the skin layers at a constant (room) temperature to reduce thermal tissue damage during MR-HIFU ablation, and accordingly, speed up the treatment procedure.

In this study we demonstrate the concept for the clinical use of a direct skin cooling (DISC) device added to an MRHIFU system during volumetric ablation of uterine fibroids. The purpose of this study was to evaluate the feasibility and safety of uterine fibroid treatments using this DISC system as additional buffer against potential adverse events related to skin heating.

\section{Materials and Methods}

2.1. Patients and Lesions. This prospective nonrandomized proof-of-concept study (NL45458.041.13) was approved by the Institutional Review Board and was conducted in accordance with the rules for international good clinical practice. Patients who participated in this study were already selected for clinical MR-HIFU ablation of uterine fibroids based on their history, physical examination, and diagnostic pelvic MRI examination. Routine inclusion and exclusion were carried out; eligible patients met the following inclusion criteria [21]: (1) 18 years or older, (2) clinically diagnosed with symptomatic uterine fibroids, (3) referred by their gynecologists with an absolute indication for intervention, (4) premenopausal or perimenopausal, (5) not currently pregnant or breastfeeding, (6) no general contraindications for magnetic resonance imaging (MRI) and MR contrast agents, and (7) able to undergo the MR-HIFU procedure based on a diagnostic pelvic MRI examination in prone position (Achieva 1.5-T, Philips Healthcare, Best, The Netherlands). Exclusion criteria were (1) the presence of other pelvic diseases, (2) unavoidable extensive scar tissue in the lower abdomen (in some cases alternate ultrasound beam paths were possible to avoid scar tissue, e.g., via beam shaping or beam angulation), (3) interposition of the bowel between the anterior abdominal wall and the dominant uterine fibroid, (4) excessive fibroid size ( $\geq 12 \mathrm{~cm})$, and (5) too many lesions $(\geq 10$ uterine fibroids). All patients gave written informed consent for conducting an MR-HIFU procedure with the presence of the direct skin cooling (DISC) device.

2.2. MR-HIFU System. All treatments were performed on a modified clinical MR-HIFU fibroid therapy system (Sonalleve, Philips Healthcare, Vantaa, Finland) integrated into a 1.5-T MRI scanner (Achieva, Philips Healthcare, Best, The Netherlands). MR images were used for treatment localization, feedback control (beam guidance), real-time temperature mapping with the proton resonance frequency shift (PRFS) thermometry method, and posttreatment verification of the ablated tissue. The curved patient MR table top incorporated a phased-array 256-channel HIFU transducer (radius of curvature: $14 \mathrm{~cm}$, operating at $1.2 \mathrm{MHz}$ ) housed in an electromechanical positioning system to deliver spatially and temporally controlled heating. The DISC device consisted of a liquid (water) cooling reservoir which was mounted on top of the standard clinical MR-HIFU table top, between the degassed liquid (oil) bath-in which the HIFU transducer is immersed-and the patients' skin. A schematic illustration of the clinical volumetric MR-HIFU system with and without the presence of the investigational DISC device is shown in Figure 1. The water cooling reservoir was connected to a water pump, cooling element, temperature regulator, degasser, and a bubble-trap to assure that air bubbles were extracted from the DISC system. The temperature of the water cooling reservoir was regulated at a constant room temperature $(T \approx$ $20^{\circ} \mathrm{C}$ ), such that the temperature was well tolerated on bare skin. The DISC system was filled with degassed water and turned on (10 minutes) before the start of the MR-HIFU treatment to establish the target temperature of the water cooling reservoir. By the active displacement of water through the liquid cooling reservoir (circuit) a stable temperature could be guaranteed. The temperatures in the water cooling reservoir were measured using a fiber-optical temperature sensor (SoftSens, Opsens Inc., Québec, QC, Canada), which was placed in the water between the two Mylar (polyethylene terephthalate) membranes outside the immediate acoustic beam path. Since the skin is in direct contact with the cooled water reservoir 


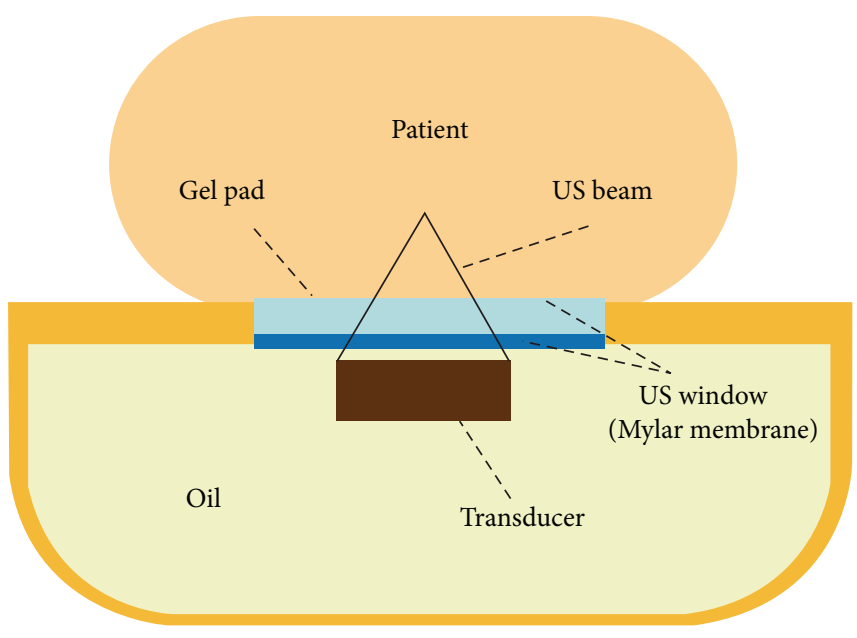

(a)

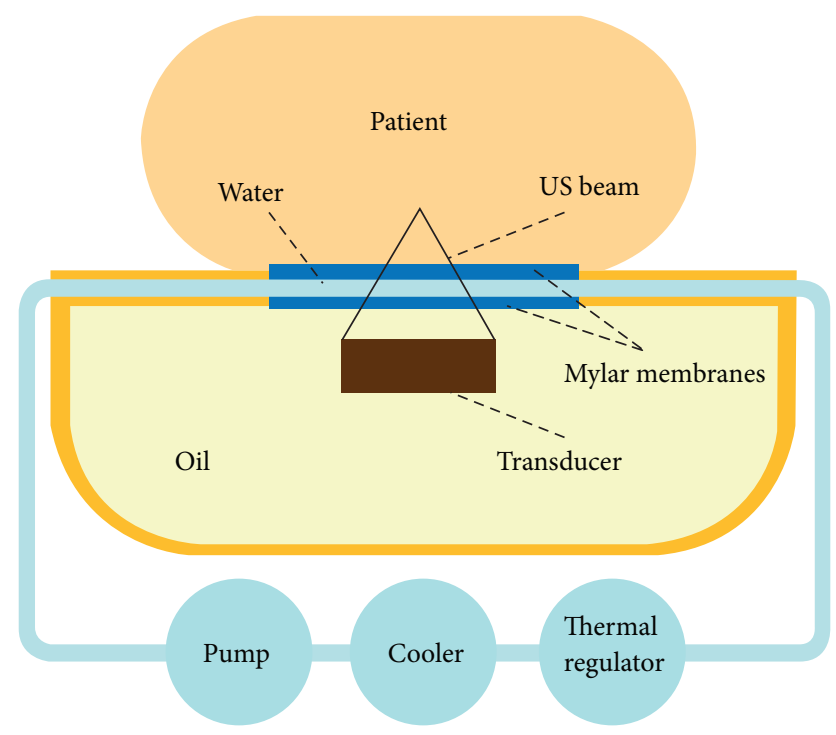

(b)

FIGURE 1: Schematic illustration of the differences between the clinical volumetric MR-HIFU system with (b) and without (a) the presence of the investigational DISC device.

(separated only by a $50 \mu \mathrm{m}$ thick membrane), the measured water temperature directly reflects the patient's skin temperature throughout the MR-HIFU ablation. Local hotspots on skin level that appear during individual sonications are equilibrated on timescales of some tens of seconds. During acquisition of diagnostic MR images, the flow within the water cooling reservoir was stopped to prevent artifacts on the MR data.

2.3. MR-HIFU Procedure. All patients were treated in an outpatient setting. Patient preparation on the day of the MRHIFU procedure (i.e., hair removal of the lower abdomen; insertion of an intravenous line and Foley catheter), treatment planning, and the volumetric ablation protocol have been described in previous publications [19, 21]. Cooling times were enforced as in normal MR-HIFU treatments, so that the DISC device was used as an additional safety buffer against potential adverse events related to skin heating. The required cooling times of at least 90 seconds were respected as suggested by the feedback MR-HIFU system. A standardized preprocedural pain management protocol was used with paracetamol 1,000 mg intravenous (Paracetamol Kabi, 10 mg/mL, Fresenius Kabi Nederland B.V., Schelle, Belgium), diclofenac $75 \mathrm{mg}$ intravenous (Voltaren, $25 \mathrm{mg} / \mathrm{mL}$, Novartis Pharma B.V., Arnhem, The Netherlands), and oxycodone $5 \mathrm{mg}$ capsules (OxyNorm, $5 \mathrm{mg}$, Mundipharma Pharmaceuticals B.V., Hoevelaken, The Netherlands). In this study, patients were asked to lie down in prone position (feet first) on the patient MR table top with the integrated DISC device. A wetted ultrasound gel pad $(7.5 \mathrm{~mm})$ or a thin mixture $(10: 1)$ of degassed water and ultrasound gel (gel film) was used as coupling agents to provide adequate direct contact for the ultrasound waves to penetrate the patients' skin. Two different types of acoustic couplers were evaluated, in order to assess whether treatment could also be carried out without the commonly used gel pad. Standard MR images were acquired to detect any obstacles in the ultrasound beam path and the contact surface to ensure that MRHIFU ablation was safe with respect to heating in unwanted locations due to the presence of air bubbles, scars, bowel, bone, and/or implants. A typical representation of the MR images in the MR-HIFU user interface is shown in Figure 2. The following MR sequences were used: coronal membrane bubble scan (three-dimensional (3D) spoiled gradient echo (FFE) with repetition time [TR], 5.8 milliseconds [ms]; echo time [TE], $4.0 \mathrm{~ms}$; flip angle [FA], $6^{\circ}$; field of view [FOV], $260 \mathrm{~mm} \times 260 \mathrm{~mm}$; acquired [ACQ] voxel size, $1.00 \times 1.00$ $\times 2.00 \mathrm{~mm}^{3}$; reconstructed [REC] voxel size, $0.49 \times 0.49 \times$ $1.00 \mathrm{~mm}^{3}$; number of averages [NSA], 6; acquisition time, 00:39 minutes), coronal skin bubble scan (multislice singleecho FFE with TR, $150 \mathrm{~ms}$; TE, $15 \mathrm{~ms}$; FA, 55 ; FOV, $280 \mathrm{~mm}$ $\times 280 \mathrm{~mm}$; ACQ voxel size, $1.25 \times 1.25 \times 2.50 \mathrm{~mm}^{3}$; REC voxel size, $0.31 \times 0.31 \times 2.50 \mathrm{~mm}^{3}$; NSA, 2 ; acquisition time, 00:38 minutes), coronal scar scan (single-echo 3D FFE with TR, $21 \mathrm{~ms}$; TE, $6.0 \mathrm{~ms}$; FA, $15^{\circ}$; FOV, $200 \mathrm{~mm} \times 200 \mathrm{~mm}$; ACQ voxel size, $0.89 \times 0.89 \times 2.00 \mathrm{~mm}^{3}$; REC voxel size, $0.31 \times 0.31 \times 1.00 \mathrm{~mm}^{3}$; NSA, 3; acquisition time, 02:06.5 minutes), and anatomical 3D T2-weighted (T2w) turbo spin echo (TSE) with TR, $1425 \mathrm{~ms}$; TE, $130 \mathrm{~ms}$; FA, 90 ; FOV, $250 \mathrm{~mm} \times 250 \mathrm{~mm}$; ACQ voxel size, $1.20 \times 1.39 \times 3.00 \mathrm{~mm}^{3}$; REC voxel size, $0.49 \times 0.49 \times 1.50 \mathrm{~mm}^{3}$; NSA, 2 ; acquisition time, 03:50.8 minutes, and 3D T1-weighted (T1w) FFE with TR, $3.6 \mathrm{~ms}$; TE, $1.90 \mathrm{~ms}$; FA, $7^{\circ}$; FOV, $220 \mathrm{~mm} \times 240 \mathrm{~mm}$; ACQ voxel size, $1.25 \times 1.53 \times 2.50 \mathrm{~mm}^{3}$; REC voxel size, $0.47 \times 0.47$ $\times 1.25 \mathrm{~mm}^{3}$; NSA, 8 ; acquisition time, 01:57.3 minutes. During the treatment procedure, patients received conscious sedation (propofol-ketamine combination) to reduce and control pain or discomfort and involuntary movements. Monitored anesthesia care (MAC) was provided by the anesthesiologist (procedural sedation specialist), which included prerocedural screening, intraprocedural support of vital functions 


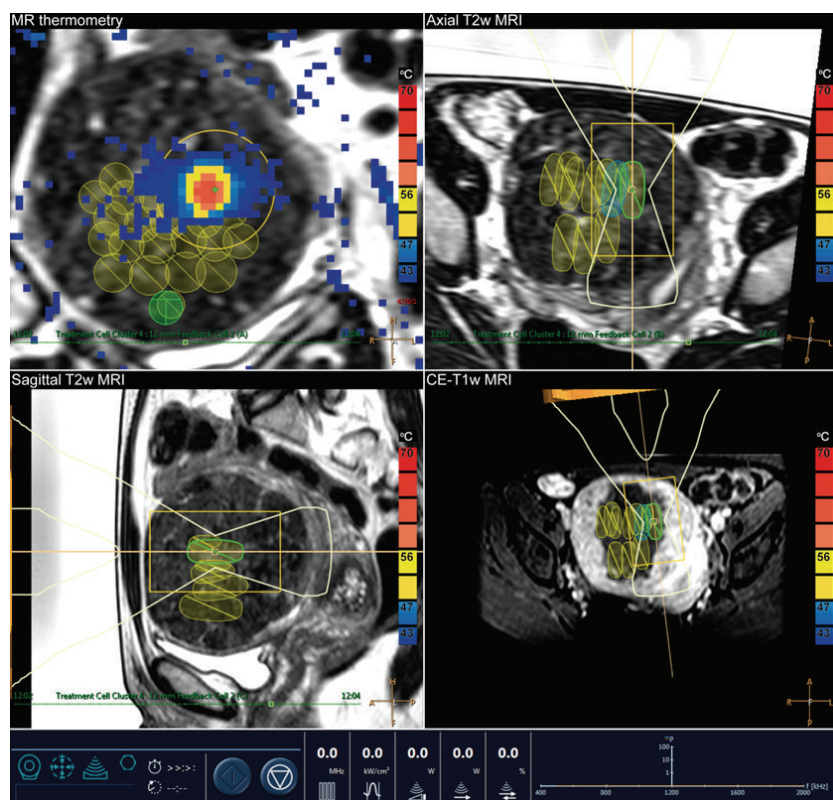

FIGURE 2: Typical representation of the MR images in the MR-HIFU user interface during treatment of uterine fibroids. The patient was lying in prone position (feet first) on the MR table top with the integrated direct skin cooling (DISC) device. The uterine fibroid was positioned directly above the MR-HIFU transducer. The ultrasound beam path was planned using T2-weighted MRI in three orthogonal planes, that is, coronal (top left), axial (top right), and sagittal (bottom left) plane. During each sonication, color temperature maps were computed by the MR-HIFU system using the proton resonance frequency shift (PRFS) thermometry method and shown on top of the anatomical images (top left). Immediately after MR-HIFU treatment, the volume that was successfully treated was defined as the nonenhancing part of the fibroid on contrast-enhanced T1weighted MRI (bottom right).

and administration of anesthetic agents, and postprocedural anesthesia management. After completion of the MRHIFU procedure, a set of MR images of the target region was obtained including a contrast-enhanced (gadobutrol, Gadovist, $0.1 \mathrm{mmol} / \mathrm{kg}$, Bayer Schering Pharma) T1-weighted (CE-T1w) TFE sequence (with TR, $5.4 \mathrm{~ms}$; TE, $2.6 \mathrm{~ms}$; FA, $10^{\circ}$; FOV, $250 \mathrm{~mm} \times 250 \mathrm{~mm}$; ACQ voxel size, $1.49 \times 1.89$ $\times 3.00 \mathrm{~mm}^{3}$; REC voxel size, $0.49 \times 0.49 \times 1.50 \mathrm{~mm}^{3}$; NSA, 4; acquisition time, 02:14.5 minutes) to allow a sum-of-slice measurement of the nonperfused volume (NPV), indicating the volume of fibroid tissue that is nonviable. Following this, patients were conducted to the recovery room for medical supervision before being discharged from the hospital on the same day.

2.4. Data Collection. The primary endpoint of this study was the technical feasibility of clinical MR-HIFU fibroid treatments with direct skin cooling, as determined by recording the successfully completed treatments using the investigational DISC device. Treatment completion was judged by an experienced operating physician (M.v.d.B.). If a treatment was aborted before the desired ablation volume was achieved and the backup CE-labelled MR-HIFU system had to be used, the treatment was counted as a failure. The extent of treatment was reported by measuring the NPV ratios, defined as the nonenhancing part of the fibroid divided by the fibroid volume. The achieved NPV ratios were compared to data from the literature to assess whether the performed treatments represent typical MR-HIFU ablations. In order to determine the effectiveness of the MR-HIFU treatments with the DISC device, the energy deposition rate (in kilojoule per hour $[\mathrm{kJ} / \mathrm{h}]$ ) was calculated by dividing the deposited treatment energy $[\mathrm{kJ}]$ by the total treatment time $(1 /[\mathrm{h}])$. The treatment time was defined as the time from the start of the first to the end of the last sonication.

The secondary endpoint of this study was to gain insights into factors influencing the safety. Safety was assessed by recording (serious) adverse events and whether they were related to the investigational DISC device, in particular by inspection of patients' skin immediately after MR-HIFU treatment. All adverse events (AEs) were recorded and classified following the 14155:2011 standard for Good Clinical Practice in clinical investigation of medical devices for human subjects issued by the International Organization for Standardization (ISO). Any AEs observed during or after MR-HIFU treatment were followed and documented until they had abated or until a stable situation had been reached. Patients were contacted by telephone 3 days, 7 days, and 30 days after the MR-HIFU procedure to determine whether any AEs had occurred. A pain assessment scale was obtained using the visual analogue scale (VAS) from 0 (no pain) to 10 (worst pain possible). The flow chart in Figure 3 shows the patients' progress through the study.

2.5. Statistical Analysis. Data were prospectively collected and analyzed to evaluate the safety and feasibility of performing MR-HIFU treatment of uterine fibroids in a volumetric MR-HIFU system equipped with a DISC device. The sample size calculation was based on the two-sided AgrestiCoull 95\% confidence interval (95\% CI) [25]. The AgrestiCoull interval is known to provide optimal coverage for binominal proportions when the sample size is small and the success probability approaches 0 or $1[26,27]$. The estimate of the Agresti-Coull 95\% CI showed that 8 successfully treated patients would be needed to detect a therapy success rate of at least $63 \%$. Estimations for different proportions of successful therapy completion are presented in Table 1. Descriptive statistics were used to describe the distribution of the patients' demographic and lesion characteristics. The treatment data of each patient was subsequently summarized and reported. Categorical data are presented in number and percentage, whereas continuous data are given in median and interquartile range (IQR). Statistical analyses were performed using IBM SPSS Statistics, version 20.0 (Armonk, New York, USA).

\section{Results}

Eight Caucasian patients with nine treatable uterine fibroids were consecutively enrolled in this study. One patient was treated for two uterine fibroids during the same MR-HIFU treatment. Three patients had a scar in the lower abdomen: 


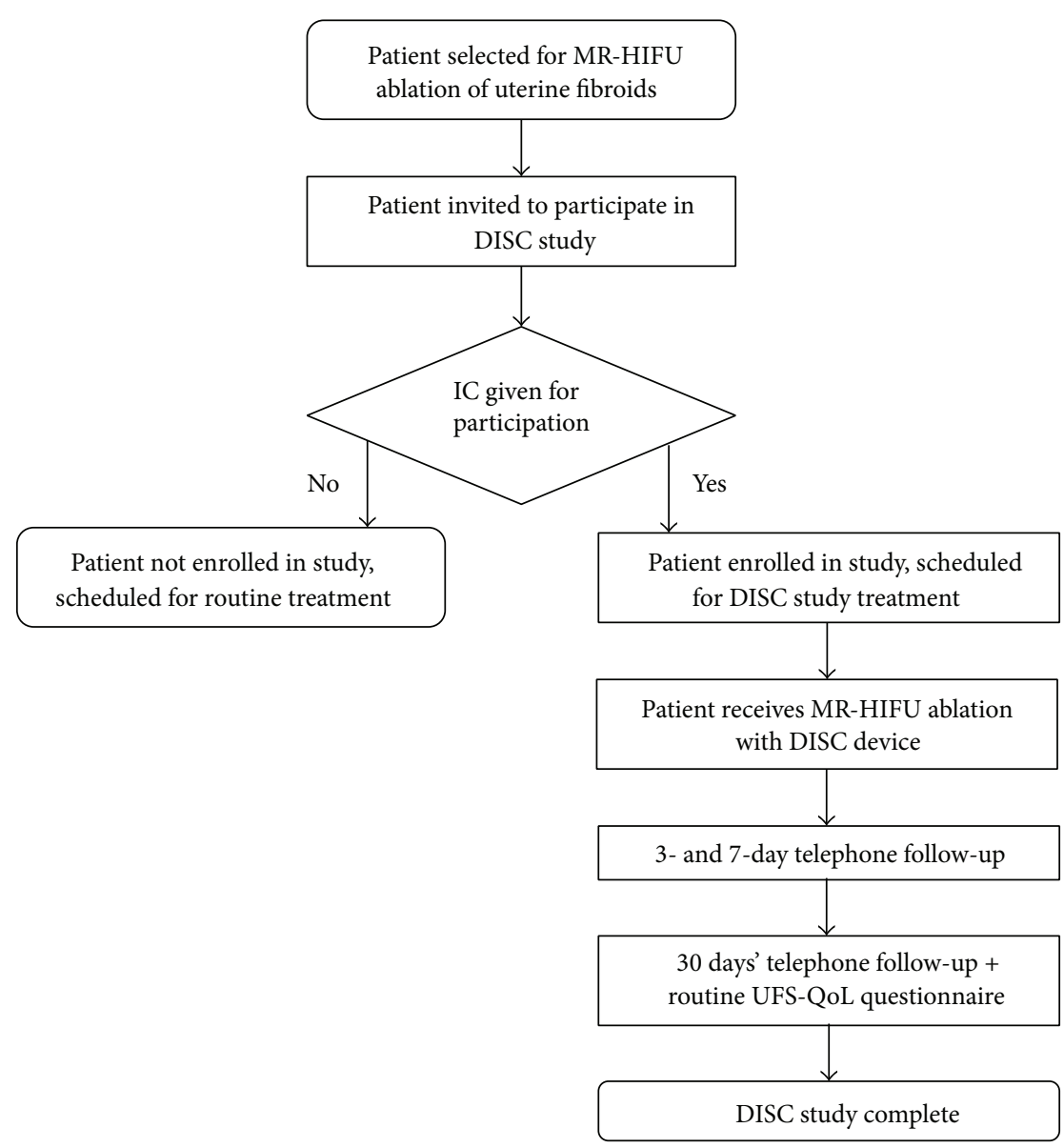

FIGURE 3: Flowchart shows the patients' progress $(n=8)$ through the DISC study. MR-HIFU: magnetic resonance-guided high-intensity focused ultrasound; DISC: direct skin cooling; IC: informed consent; UFS-QoL: uterine fibroid symptom and health-related quality of life.

TABLE 1: Estimated binominal proportion confidence intervals using the Agresti-Coull (adjusted Wald) method [25].

\begin{tabular}{|c|c|c|c|c|c|}
\hline \multirow{3}{*}{ Sample size ${ }^{\mathrm{a}}$} & \multicolumn{2}{|c|}{ Observed outcome } & \multicolumn{3}{|c|}{ Estimation } \\
\hline & \multirow{2}{*}{ Successes $^{\mathrm{a}}$} & \multirow{2}{*}{ Failures $^{\mathrm{a}}$} & \multirow{2}{*}{$\widehat{p}$} & \multicolumn{2}{|c|}{$95 \%-\mathrm{CI}$} \\
\hline & & & & Lower & Upper \\
\hline 8 & 8 & 0 & 0.84 & 0.63 & 1.00 \\
\hline 8 & 7 & 1 & 0.75 & 0.51 & 1.00 \\
\hline 8 & 6 & 2 & 0.67 & 0.40 & 0.94 \\
\hline 8 & 5 & 3 & 0.58 & 0.30 & 0.87 \\
\hline
\end{tabular}

${ }^{\mathrm{a}}$ Values are expressed in numbers; $\widehat{p}$ : sample proportion of success; CI: confidence interval.

one patient (ID number 4) had undergone an abdominal myomectomy (Pfannenstiel incision), one patient (ID number 1) underwent an open appendectomy (McBurney incision), and one patient (ID number 2) had minor laparoscopic scars after a diagnostic laparoscopy due to chronic abdominal pain. In patient 4, urinary bladder filling (with a saline solution) was used to avoid the surgical scar in the ultrasound beam path. Baseline data collected for each patient at the beginning of the study are presented in Table 2. All MR-HIFU treatments were successfully completed using the investigational DISC device; no technical failure was observed. The use of the backup CE-labeled MR-HIFU system was not necessary. The median treatment time was 192 minutes (IQR
[180-225]), with a median energy deposition rate of $67 \mathrm{~kJ} / \mathrm{h}$ (IQR [55-92]). The median volume of the uterine fibroids was $147 \mathrm{~cm}^{3}$ (IQR [49-338]); the median maximum fibroid diameter was $7.7 \mathrm{~cm}$ (IQR [5.1-8.8]). A median nonperfused volume of $56 \mathrm{~cm}^{3}$ (IQR [14-91]) was achieved, and a median NPV ratio of 0.56 (IQR [0.27-0.72]) was found. In one patient (ID number 7), no NPV could be achieved due to insufficient heating probably as a result of the tissue characteristics of the uterine fibroid (type 3) [28]. Table 3 shows an overview of the treatment data of each patient treated in this study.

No serious (device-related) adverse events were observed within 30 days' follow-up. No patient required prolonged observation before discharge or readmission after hospital 
TABLE 2: Baseline characteristics and MRI findings $(n=8)$.

\begin{tabular}{|c|c|}
\hline \multicolumn{2}{|c|}{ Patient characteristics } \\
\hline Age (years) ${ }^{\mathrm{a}}$ & $42.5(38.8-47.8)$ \\
\hline BMI $\left(\mathrm{kg} / \mathrm{m}^{2}\right)^{\mathrm{a}}$ & $23.1(19.9-28.1)$ \\
\hline \multicolumn{2}{|l|}{ Symptoms ${ }^{\mathrm{b}}$} \\
\hline Menorrhagia & $3(38 \%)$ \\
\hline Bulky symptoms & $4(50 \%)$ \\
\hline Infertility & $1(12 \%)$ \\
\hline $\mathrm{tSSS}^{\mathrm{a}}$ & $57.9(38.3-79.0)$ \\
\hline Total HRQoL ${ }^{\mathrm{a}}$ & $48.7(28.9-80.4)$ \\
\hline \multicolumn{2}{|c|}{ Lesion characteristics } \\
\hline \multicolumn{2}{|l|}{ Number of fibroids ${ }^{b}$} \\
\hline Solitary fibroid & $2(25 \%)$ \\
\hline Multiple fibroids & $6(75 \%)$ \\
\hline 2-5 fibroids & $4(67 \%)$ \\
\hline 6-10 fibroids & $2(33 \%)$ \\
\hline \multicolumn{2}{|l|}{ Location of fibroids ${ }^{\mathrm{b}}$} \\
\hline Intramural & $6(67 \%)$ \\
\hline Submucosal & $3(33 \%)$ \\
\hline \multicolumn{2}{|l|}{ Type of fibroid ${ }^{b}$} \\
\hline Type 1 & $4(45 \%)$ \\
\hline Type 2 & $3(33 \%)$ \\
\hline Type 3 & $2(22 \%)$ \\
\hline Maximum fibroid diameter $(\mathrm{cm})^{\mathrm{a}}$ & $7.7(5.1-8.8)$ \\
\hline Uterine fibroid volume $\left(\mathrm{cm}^{3}\right)^{\mathrm{a}}$ & $147(49-338)$ \\
\hline
\end{tabular}

${ }^{a}$ Median (interquartile range); ${ }^{b}$ number (percentage); MR-HIFU: MRguided high-intensity focused ultrasound; tSSS: transformed symptom severity score (range 0-100); high scores indicate more severe symptoms; HRQoL: health-related quality of life (range 0-100); high scores indicate better quality of life; type 1: low signal intensity on T2-weighted imaging; type 2: intermediate signal intensity on T2-weighted imaging; type 3: high signal intensity on T2-weighted imaging; NPV: nonperfused volume.

discharge. In particular, no skin burns, cold injuries, or subcutaneous edema (determined by increased signal intensity on T2w images) was observed in patients treated with the DISC device. Several mild AEs were reported, such as abdominal pain or cramping $(n=3)$, back pain $(n=$ $3)$, abdominal tenderness $(n=2)$, ergonomic problems $(n=2)$, namely, fibular compression neuropathy due to external pressure at the right fibular head during a long treatment procedure of 230 minutes (ID number 3) and pressure marks due to the curved patient table top, dyspepsia and constipation $(n=2)$, dizziness $(n=3)$, and lethargy $(n=4)$. One mild device-related AE occurred, namely, coldness-related discomfort which was rapidly resolved with a hot water bottle on the day of treatment $(n=2)$. At the moment of hospital discharge, the median VAS score was 1.5 (IQR [0.25-3.0], range [0-7]). Typically, AEs resolved within 7 days' follow-up and patients were able to resume their daily activities again. However, after 30 days, two patients were still recovering from ongoing AEs (constipation and lethargy), both probably related to the administration of anaesthetic agents.

\section{Discussion}

During MR-HIFU treatments, undesired heating outside of the targeted ablation area may occur, in particular in the near field region of the HIFU beam. Currently, the risk of near field damage is mitigated by the time-consuming enforcement of cooling periods between ultrasound sonications. Introduction of a cooled interface which allows direct cooling of the patients' skin may further mitigate undesired heating (by shifting the baseline temperature), potentially increasing treatment efficacy and providing an additional buffer. In this proof-of-concept study we have demonstrated the safety and feasibility of using a direct skin cooling (DISC) device added to a volumetric MR-HIFU system for uterine fibroid treatments. To the best of our knowledge, this concept had not yet been investigated in a clinical setting. Our results showed that it is technically feasible and safe to complete an MR-HIFU treatment with a DISC device. No thermal damage to the near field, due to temperature increase of the ultrasound energy emitted from the HIFU transducer, was observed in this study. All eight patients (100\%) could be treated as in normal clinical practice, representing a typical case series of uterine fibroid MR-HIFU treatments. No clinical or technical problems have occurred preventing MRHIFU treatments using the DISC device to be successfully completed. No serious (device-related) AEs occurred after treatment and the achieved median NPV ratio (0.56 (IQR [0.27-0.72]) was comparable to data previously published. Reported NPV ratios ranged from 0.40 to 0.70 with a median NPV ratio of 0.56 [21, 29-33]. Specifically, no skin redness and/or irritation of the abdominal wall-either related to skin heating or skin cooling-were seen. As known, cutaneous microvascular reactivity (namely, thermoregulatory reflex) is essential to maintain the human core temperature during challenges to thermal hemostasis [34, 35]. Prolonged exposure of direct cooling of the skin will eventually lead to cutaneous vasoconstriction [36-38], which may induce ischemia. Extended exposure to localized cold-induced vasoconstriction may cause various injuries to the patients' skin that typically fall within the domain termed nonfreezing cold injury (NFCI) [39, 40]. However, these injuries (e.g., chilblains or immersion foot) are most commonly reported to the body's lower extremities, such as the legs and feet [41, 42]. In addition, the literature evidence indicates that no serious thermal tissue damage is to be expected within several hours until freezing occurs $[43,44]$. In general, NFCI occurs at temperatures of $0^{\circ} \mathrm{C}$ to $15^{\circ} \mathrm{C}$. Considering the temperatures and exposure times relevant for patients during an MRHIFU procedure, it can be concluded that the probability of occurrence for this specific low temperature event was indeed very unlikely.

It should be noted that this is a technical feasibility study with a small number of patients $(n=8)$. Despite the promising preliminary results, the current study was not designed to measure the efficacy of the cooling system. Therefore, further research is needed to assess the clinical efficacy of this investigational DISC device as add-on to the volumetric MR-HIFU system. In a future step, a clinical 


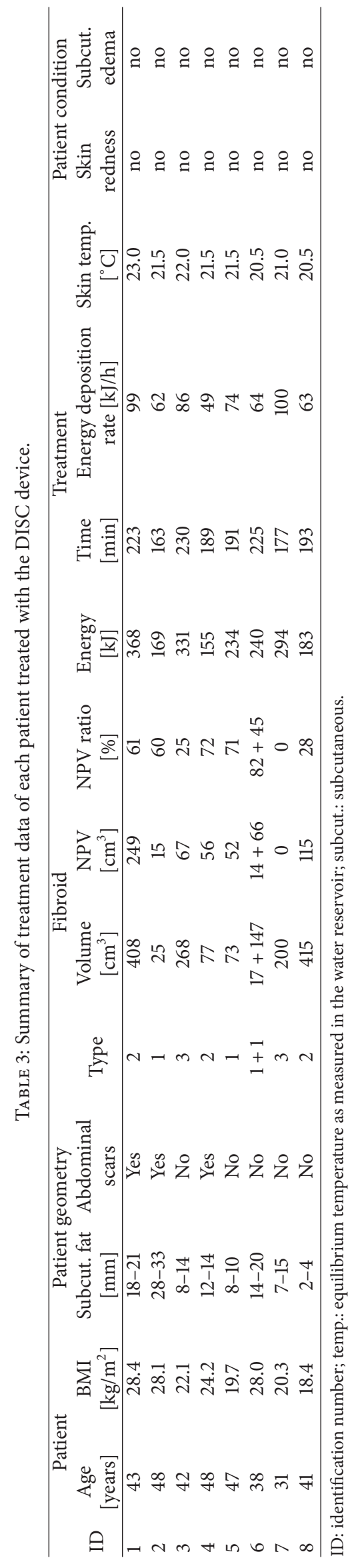


follow-up study will investigate to which extent the DISC system allows for speeding up uterine fibroid MR-HIFU treatments by benefiting from reduced cooling times. After each sonication, the cooling effect in the subcutaneous tissue layers will be systematically monitored using the T2-based thermometry as recently described by Baron et al. [45]. The acquired temperature (cool-down) information in the near field can be used to provide thermal feedback to the MRHIFU system and use this to adjust the cooling periods between the subsequent energy depositions in real time. This novel technique may result in some advantages for the patient and their treating physician: (1) ablation of larger volumes of (fibroid) tissue in the shortest period of time that is safely possible and (2) more efficient treatment of type 3 uterine fibroids with a high signal intensity on T2w imaging $[28,46]$. The fact that it is technically feasible to produce thermal lesions safely in the present study highlights the potential of this DISC system in the future MR-HIFU ablations of uterine fibroids. Another practical advantage of using DISC is that it seems sufficient to use a gel film (water-gel mixture) for acoustic coupling between the table top membrane and the depilated skin of the patient. Previously, it was easy to trap air bubbles between the commonly used $(15 \mathrm{~mm})$ gel pad and the patients' skin in the process of patient positioning. Since air bubbles may reflect the ultrasound energy_due to their low acoustic impedance [47] - they can cause abdominal pain or discomfort and skin burns. This restriction appears alleviated now by the use of a gel film, because we did not observe any air bubbles after positioning. Furthermore, without the insulating gel pad, the patient profits directly from the cooling effect without dissipation. Finally, the DISC system with gelfilm has the same thickness as the old gel pads (Figure 1). This means that it is possible to reach targets as deep in the patient body as before. Consequently, the use of a gel film will potentially reduce risks and offers opportunities to speed up the patient preparation and overall treatment time.

Several limitations to the present study need to be acknowledged. First, we did not analyze the patient-reported outcomes of symptom severity (tSSS) and health-related quality of life (HRQoL) as measure of the treatment effect. The focus of the current study was to verify that the use of the DISC device did not affect the performance of the volumetric MR-HIFU system. The upcoming studies will require a larger patient cohort, larger ablation volumes, longer follow-up, and careful evaluation of the treatment efficacy using this DISC system. This information could be used to develop targeted interventions aimed at specific subgroups of patients, such as patients with hyperintense uterine fibroids on pretreatment T2w MRI [28] with the use of targeted vessel ablation [48]. Another limitation of our study is that we did the postdischarge follow-up by telephone interviewing. While this may not give us always an objective evaluation (due to absence of nonverbal cues), it provided us with the required information about the safety outcome.

In conclusion, in this proof-of-concept study we successfully performed clinical volumetric MR-HIFU ablation of uterine fibroids using an additional DISC device for reducing the risk of thermal damage to the abdominal wall. On the basis of the small number of patients studied, using the additional DISC device in this trial appears to be safe. No serious (device-related) adverse events occurred.

\section{Conflict of Interests}

The authors declare that there is no conflict of interests regarding the publication of this paper.

\section{Acknowledgments}

This work was supported in part by Philips Healthcare (Philips Medical Systems MR, Finland). One author (Gerald Schubert) is an employee of Philips Healthcare; however the first two authors (Marlijne E. Ikink and Johanna M. M. van Breugel) in control of the study data and data analysis are not employees of Philips Healthcare.

\section{References}

[1] C. M. Tempany, E. A. Stewart, N. McDannold, B. J. Quade, F. A. Jolesz, and K. Hynynen, "MR imaging-guided focused ultrasound surgery of uterine leiomyomas: a feasibility study," Radiology, vol. 226, no. 3, pp. 897-905, 2003.

[2] S. F. Cramer and A. Patel, "The frequency of uterine leiomyomas," The American Journal of Clinical Pathology, vol. 94, no. 4, pp. 435-438, 1990.

[3] V. C. Buttram Jr. and R. C. Reiter, "Uterine leiomyomata: etiology, symptomatology, and management," Fertility and Sterility, vol. 36, no. 4, pp. 433-445, 1981.

[4] M. O. Köhler, C. Mougenot, B. Quesson et al., "Volumetric HIFU ablation under 3D guidance of rapid MRI thermometry," Medical Physics, vol. 36, no. 8, pp. 3521-3535, 2009.

[5] X. Fan and K. Hynynen, "Ultrasound surgery using multiple sonications: treatment time considerations," Ultrasound in Medicine and Biology, vol. 22, no. 4, pp. 471-482, 1996.

[6] F. C. Henriques and A. R. Moritz, "Studies of thermal injury: I. The conduction of heat to and through skin and the temperatures attained therein. A theoretical and an experimental investigation," American Journal of Pathology, vol. 23, pp. 530549, 1947.

[7] G. S. Anderson, "Human morphology and temperature regulation," International Journal of Biometeorology, vol. 43, no. 3, pp. 99-109, 1999.

[8] D. L. Kellogg and P. Pérgola, "Skin responses to exercise and training," in Exercise and Sport Science, W. E. Garrett and D. T. Kirkendall, Eds., pp. 239-250, Lippincott Williams \& Wilkins, Philadelphia, Pa, USA, 1st edition, 2000.

[9] M. L. Cohen, "Measurement of the thermal properties of human skin. A review," Journal of Investigative Dermatology, vol. 69, no. 3, pp. 333-338, 1977.

[10] M. Rowland and T. N. Tozer, "Membranes and distribution table 4-4," in Clinical Pharmacokinetics and Pharmacodynamics: Concepts and Applications, pp. 73-110, Wolters Kluwer Health/Lippincott William \& Wilkins, Philadelphia, Pa, USA, 4th edition, 2010.

[11] I. Heinonen, J. Kemppainen, K. Kaskinoro, J. Knuuti, R. Boushel, and K. K. Kalliokoski, "Capacity and hypoxic response of subcutaneous adipose tissue blood flow in humans," Circulation Journal, vol. 78, no. 6, pp. 1501-1506, 2014. 
[12] J. M. Johnson, G. L. Brengelmann, J. R. S. Hales, P. M. Vanhoutte, and C. B. Wenger, "Regulation of the cutaneous circulation," Federation Proceedings, vol. 45, no. 13, pp. 2841-2850, 1986.

[13] R. Williams, "Production and transmission of ultrasound," Physiotherapy, vol. 73, no. 3, pp. 113-116, 1987.

[14] E. A. Stewart, W. M. W. Gedroyc, C. M. C. Tempany et al., "Focused ultrasound treatment of uterine fibroid tumors: safety and feasibility of a noninvasive thermoablative technique," The American Journal of Obstetrics and Gynecology, vol. 189, no. 1, pp. 48-54, 2003.

[15] E. A. Stewart, J. Rabinovici, C. M. C. Tempany et al., "Clinical outcomes of focused ultrasound surgery for the treatment of uterine fibroids," Fertility and Sterility, vol. 85, no. 1, pp. 22-29, 2006.

[16] J. Rabinovici, Y. Inbar, A. Revel et al., "Clinical improvement and shrinkage of uterine fibroids after thermal ablation by magnetic resonance-guided focused ultrasound surgery," Ultrasound in Obstetrics \& Gynecology, vol. 30, no. 5, pp. 771-777, 2007.

[17] J. Leon-Villapalos, M. Kaniorou-Larai, and P. Dziewulski, "Full thickness abdominal burn following magnetic resonance guided focused ultrasound therapy," Burns, vol. 31, no. 8, pp. 1054-1055, 2005.

[18] G. K. Hesley, K. R. Gorny, T. L. Henrichsen, D. A. Woodrum, and D. L. Brown, "A clinical review of focused ultrasound ablation with magnetic resonance guidance: an option for treating uterine fibroids," Ultrasound Quarterly, vol. 24, no. 2, pp. 131139, 2008.

[19] M. J. Voogt, H. Trillaud, Y. S. Kim et al., "Volumetric feedback ablation of uterine fibroids using magnetic resonance-guided high intensity focused ultrasound therapy," European Radiology, vol. 22, no. 2, pp. 411-417, 2012.

[20] R. MacHtinger, Y. Inbar, S. Cohen-Eylon, D. Admon, A. AlagemMizrachi, and J. Rabinovici, "MR-guided focus ultrasound (MRgFUS) for symptomatic uterine fibroids: predictors of treatment success," Human Reproduction, vol. 27, no. 12, pp. 3425-3431, 2012.

[21] M. E. Ikink, M. J. Voogt, H. M. Verkooijen et al., "Mid-term clinical efficacy of a volumetric magnetic resonance-guided high-intensity focused ultrasound technique for treatment of symptomatic uterine fibroids," European Radiology, vol. 23, no. 11, pp. 3054-3061, 2013.

[22] C. Damianou and K. Hynynen, "Focal spacing and near-field heating during pulsed high temperature ultrasound therapy," Ultrasound in Medicine and Biology, vol. 19, no. 9, pp. 777-787, 1993.

[23] K. Hynynen, A. Darkazanli, E. Unger, and J. F. Schenck, "MRIguided noninvasive ultrasound surgery," Medical Physics, vol. 20, no. 1, pp. 107-115, 1993.

[24] Y.-S. Kim, B. Keserci, A. Partanen et al., "Volumetric MRHIFU ablation of uterine fibroids: role of treatment cell size in the improvement of energy efficiency," European Journal of Radiology, vol. 81, no. 11, pp. 3652-3659, 2012.

[25] A. Agresti and B. A. Coull, "Approximate is better than "exact" for interval estimation of binomial proportions," The American Statistician, vol. 52, no. 2, pp. 119-126, 1998.

[26] D. L. Brown, T. T. Cai, and A. DasGupta, "Confidence intervals for a binomial proportion and asymptotic expansions," The Annals of Statistics, vol. 30, no. 1, pp. 160-201, 2002.

[27] J. Sauro and J. R. Lewis, "Estimating completion rates from small samples using binominal confidence intervals: comparisons and recommendations," in Proceedings of the Human Factors and
Ergonomics Society Annual Meeting, vol. 49, pp. 2100-2103, 2005.

[28] K. Funaki, H. Fukunishi, T. Funaki, K. Sawada, Y. Kaji, and T. Maruo, "Magnetic resonance-guided focused ultrasound surgery for uterine fibroids: relationship between the therapeutic effects and signal intensity of preexisting T2-weighted magnetic resonance images," The American Journal of Obstetrics and Gynecology, vol. 196, no. 2, pp. e184-e186, 2007.

[29] M. J. Park, Y.-S. Kim, H. Rhim, and H. K. Lim, "Safety and therapeutic efficacy of complete or near-complete ablation of symptomatic uterine fibroid tumors by MR imaging-guided high-intensity focused US Therapy," Journal of Vascular and Interventional Radiology, vol. 25, no. 2, pp. 231-239, 2014.

[30] Y.-S. Kim, M. J. Park, B. Keserci et al., "Uterine fibroids: postsonication temperature decay rate enables prediction of therapeutic responses to MR imaging-guided high-lntensity focused ultrasound ablation," Radiology, vol. 270, no. 2, pp. 589600, 2014.

[31] S.-W. Yoon, S. H. Cha, Y. G. Ji, H. C. Kim, M. H. Lee, and J. H. Cho, "Magnetic resonance imaging-guided focused ultrasound surgery for symptomatic uterine fibroids: estimation of treatment efficacy using thermal dose calculations," European Journal of Obstetrics Gynecology \& Reproductive Biology, vol. 169, no. 2, pp. 304-308, 2013.

[32] M. J. Park, Y.-S. Kim, B. Keserci, H. Rhim, and H. K. Lim, "Volumetric MR-guided high-intensity focused ultrasound ablation of uterine fibroids: treatment speed and factors influencing speed," European Radiology, vol. 23, no. 4, pp. 943-950, 2013.

[33] S.-W. Yoon, S. J. Seong, S.-G. Jung, S. Y. Lee, H. S. Jun, and J. T. Lee, "Mitigation of abdominal scars during MR-guided focused ultrasound treatment of uterine leiomyomas with the use of an energy-blocking scar patch," Journal of Vascular and Interventional Radiology, vol. 22, no. 12, pp. 1747-1750, 2011.

[34] N. Charkoudian, "Skin blood flow in adult human thermoregulation: how it works, when it does not, and why," Mayo Clinic Proceedings, vol. 78, no. 5, pp. 603-612, 2003.

[35] J. M. Johnson and D. L. Kellogg Jr., "Thermoregulatory and thermal control in the human cutaneous circulation," Frontiers in Bioscience-Scholar, vol. 2, no. 3, pp. 825-853, 2010.

[36] S. Khoshnevis, N. K. Craik, and K. R. Diller, "Cold-induced vasoconstriction may persist long after cooling ends: an evaluation of multiple cryotherapy units," Knee Surgery, Sports Traumatology, Arthroscopy, 2014.

[37] G. J. Hodges, K. Zhao, W. A. Kosiba, and J. M. Johnson, “The involvement of nitric oxide in the cutaneous vasoconstrictor response to local cooling in humans," Journal of Physiology, vol. 574, no. 3, pp. 849-857, 2006.

[38] C. T. Minson, "Thermal provocation to evaluate microvascular reactivity in human skin," Journal of Applied Physiology, vol. 109, no. 4, pp. 1239-1246, 2010.

[39] C. H. E. Imray, P. Richards, J. Greeves, and J. W. Castellani, "Nonfreezing cold-induced injuries," Journal of the Royal Army Medical Corps, vol. 157, no. 1, pp. 79-84, 2011.

[40] W. B. Long III, R. F. Edlich, K. L. Winters, and L. D. Britt, "Cold injuries," Journal of Long-Term Effects of Medical Implants, vol. 15, no. 1, pp. 67-78, 2005.

[41] B. J. Ingram and T. J. Raymond, "Recognition and treatment of freezing and nonfreezing cold injuries," Current Sports Medicine Reports, vol. 12, no. 2, pp. 125-130, 2013.

[42] R. Sallis and C. M. Chassay, "Recognizing and treating common cold-induced injury in outdoor sports," Medicine \& Science in Sports \& Exercise, vol. 31, no. 10, pp. 1367-1373, 1999. 
[43] J. A. McMahon and A. Howe, "Cold weather issues in sideline and event management," Current Sports Medicine Reports, vol. 11, no. 3, pp. 135-141, 2012.

[44] B. E. Tlougan, A. J. Mancini, J. A. Mandell, D. E. Cohen, and M. R. Sanchez, "Skin conditions in figure skaters, ice-hockey players and speed skaters: part II cold-induced, infectious and inflammatory dermatoses," Sports Medicine, vol. 41, no. 11, pp. 967-984, 2011.

[45] P. Baron, M. Ries, R. Deckers et al., "In vivo $\mathrm{T}_{2}$-based MR thermometry in adipose tissue layers for high-intensity focused ultrasound near-field monitoring," Magnetic Resonance in Medicine, vol. 72, no. 4, pp. 1057-1064, 2013.

[46] Z. M. Lénárd, N. J. McDannold, F. M. Fennessy et al., "Uterine leiomyomas: MR imaging-guided focused ultrasound surgeryimaging predictors of success 1," Radiology, vol. 249, no. 1, pp. 187-194, 2008.

[47] P. N. T. Wells, "Absorption and dispersion of ultrasound in biological tissue," Ultrasound in Medicine \& Biology, vol. 1, no. 4, pp. 369-376, 1975.

[48] M. J. Voogt, M. Van Stralen, M. E. Ikink et al., "Targeted vessel ablation for more efficient magnetic resonance-guided highintensity focused ultrasound ablation of uterine fibroids," CardioVascular and Interventional Radiology, vol. 35, no. 5, pp. 1205-1210, 2012. 


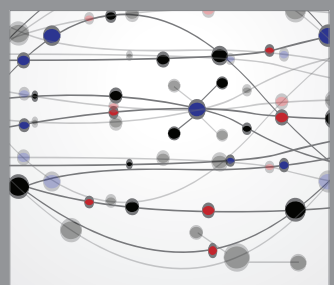

The Scientific World Journal
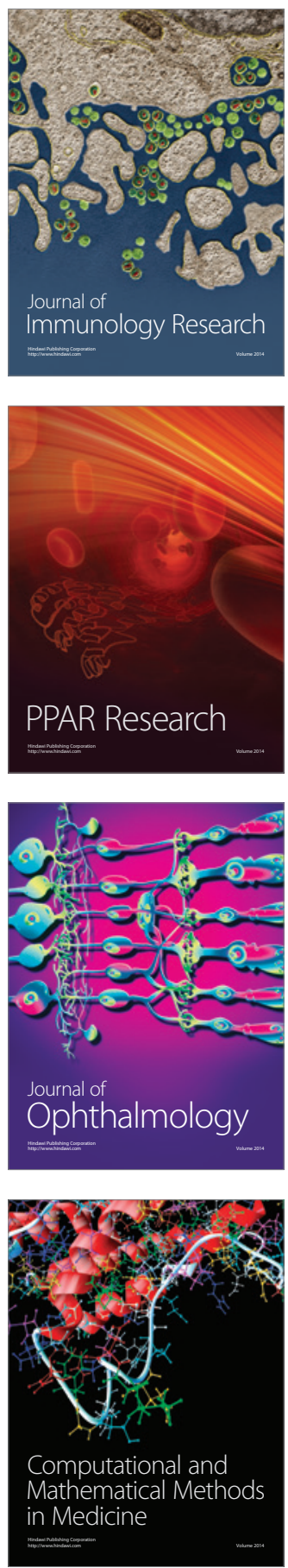

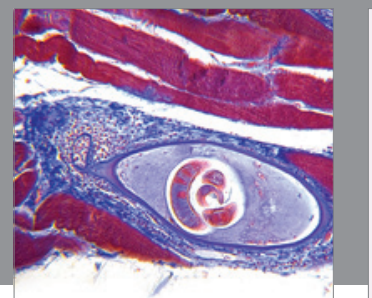

Gastroenterology

Research and Practice
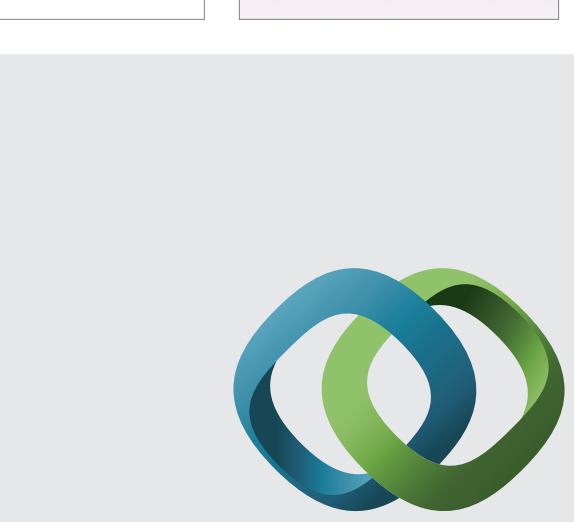

\section{Hindawi}

Submit your manuscripts at

http://www.hindawi.com
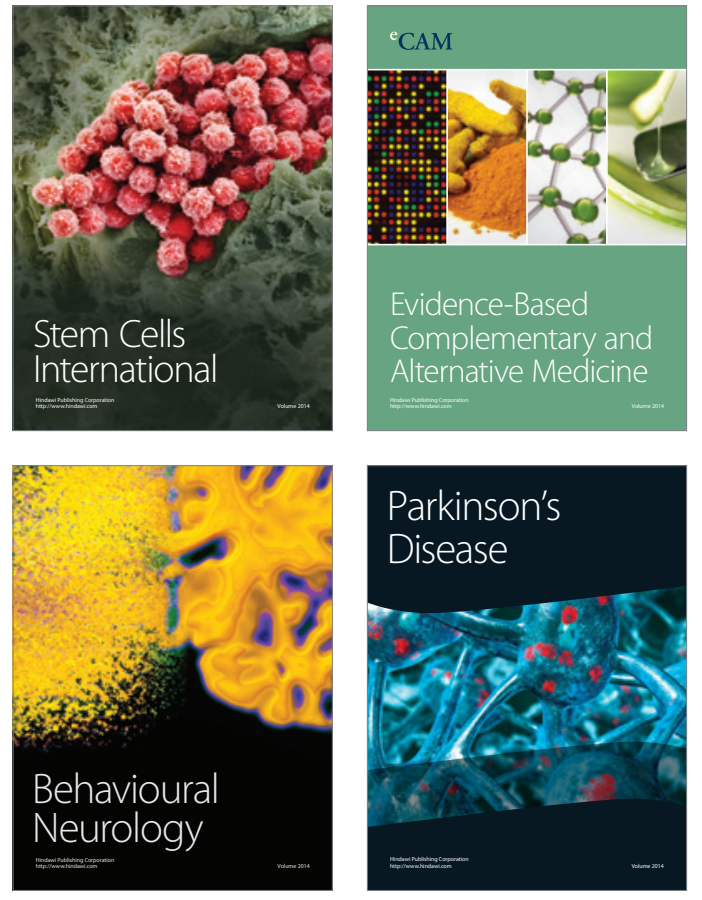
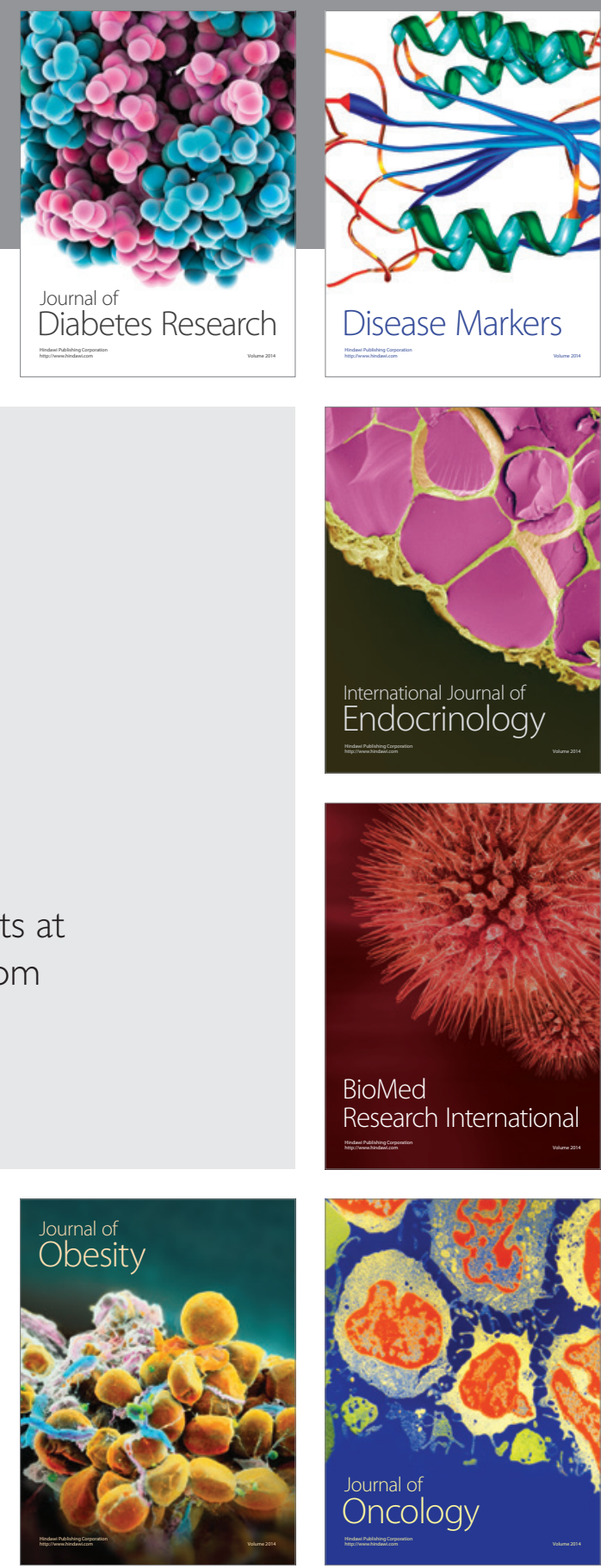

Disease Markers
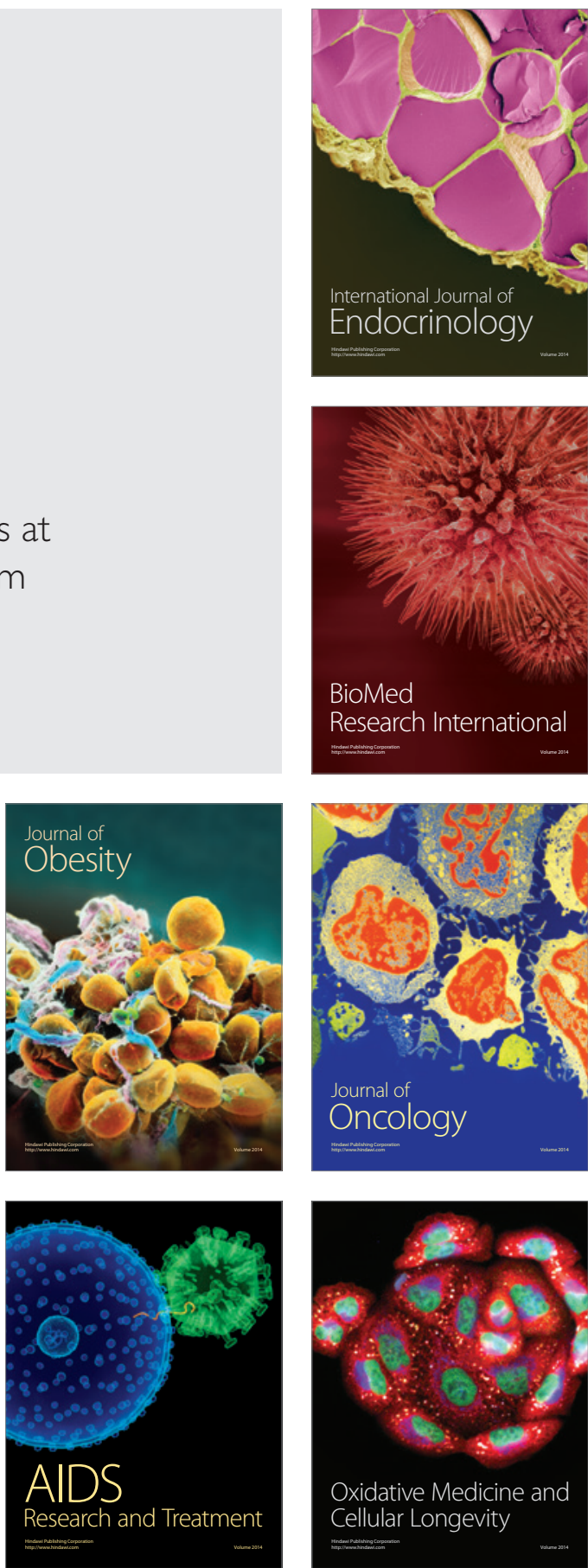